\title{
Multiple approaches for massively parallel sequencing of SARS-CoV-2 genomes directly from clinical samples
}

Minfeng Xiao ${ }^{1,2+}$, Xiaoqing Liü ${ }^{3 \dagger}$, Jingkai $\mathrm{Ji}^{1,2,4+}$, Min $\mathrm{Li}^{1,2,5 \dagger}$, Jiandong $\mathrm{Li}^{1,2,5+}$, Lin Yang ${ }^{6 \dagger}$, Wanying Sun ${ }^{1,2,5}$, Peidi Ren ${ }^{1,2}$, Guifang Yang ${ }^{6}$, Jincun Zhao ${ }^{3,7}$, Tianzhu Liang ${ }^{1,2}$, Huahui Ren', Tian Chen', Huanzi Zhong ', Wenchen Song ${ }^{1,2}$, Yanqun Wang ${ }^{3}$, Ziqing Deng ${ }^{1,2}$, Yanping Zhao ${ }^{1,2}$, Zhihua Ou ${ }^{1,2}$, Daxi Wang ${ }^{1,2}$, Jielun Cai ${ }^{1}$, Xinyi Cheng ${ }^{1,2,8}$, Taiqing Feng ${ }^{6}$, Honglong Wu ${ }^{9}$, Yanping Gong ${ }^{9}$, Huanming Yang ${ }^{1,10}$, Jian Wang ${ }^{1,10}$, Xun X X $u^{1,11}$, Shida Zhu ${ }^{1,12}$, Fang Chen ${ }^{1,6}$, Yanyan Zhang ${ }^{6^{*}+}$, Weijun Chen ${ }^{5,9^{* \dagger}}$, Yimin Li ${ }^{3^{* \dagger}}$ and Junhua $\mathrm{Li}^{1,2,8^{* \dagger}}$ (D)

\begin{abstract}
Background: COVID-19 (coronavirus disease 2019) has caused a major epidemic worldwide; however, much is yet to be known about the epidemiology and evolution of the virus partly due to the scarcity of full-length SARS-CoV-2 (severe acute respiratory syndrome coronavirus 2) genomes reported. One reason is that the challenges underneath sequencing SARS-CoV-2 directly from clinical samples have not been completely tackled, i.e., sequencing samples with low viral load often results in insufficient viral reads for analyses.

Methods: We applied a novel multiplex PCR amplicon (amplicon)-based and hybrid capture (capture)-based sequencing, as well as ultra-high-throughput metatranscriptomic (meta) sequencing in retrieving complete genomes, inter-individual and intra-individual variations of SARS-CoV-2 from serials dilutions of a cultured isolate, and eight clinical samples covering a range of sample types and viral loads. We also examined and compared the sensitivity, accuracy, and other characteristics of these approaches in a comprehensive manner.
\end{abstract}

(Continued on next page)

\footnotetext{
*Correspondence: zhangyanyan@genomics.cn; chenwj@bgi.com;

dryiminli@vip.163.com; lijunhua@genomics.cn

${ }^{\dagger}$ Minfeng Xiao, Xiaoqing Liu, Jingkai Ji, Min Li, Jiandong Li, and Lin Yang are the joint first authors.

'Yanyan Zhang, Weijun Chen, Yimin Li, and Junhua Li are the joint senior authors.

${ }^{6} \mathrm{MGl}, \mathrm{BGI}-$ Shenzhen, Shenzhen 518083, China

${ }^{5}$ BGI Education Center, University of Chinese Academy of Sciences, Shenzhen 518083, China

${ }^{3}$ State Key Laboratory of Respiratory Disease, National Clinical Research Center for Respiratory Disease, Guangzhou Institute of Respiratory Health, the First Affiliated Hospital of Guangzhou Medical University, Guangzhou, China 'BGl-Shenzhen, Shenzhen 518083, China

Full list of author information is available at the end of the article
}

(c) The Author(s). 2020 Open Access This article is licensed under a Creative Commons Attribution 4.0 International License, which permits use, sharing, adaptation, distribution and reproduction in any medium or format, as long as you give appropriate credit to the original author(s) and the source, provide a link to the Creative Commons licence, and indicate if changes were made. The images or other third party material in this article are included in the article's Creative Commons licence, unless indicated otherwise in a credit line to the material. If material is not included in the article's Creative Commons licence and your intended use is not permitted by statutory regulation or exceeds the permitted use, you will need to obtain permission directly from the copyright holder. To view a copy of this licence, visit http://creativecommons.org/licenses/by/4.0/ The Creative Commons Public Domain Dedication waiver (http://creativecommons.org/publicdomain/zero/1.0/) applies to the data made available in this article, unless otherwise stated in a credit line to the data. 
(Continued from previous page)

Results: We demonstrated that both amplicon and capture methods efficiently enriched SARS-CoV-2 content from clinical samples, while the enrichment efficiency of amplicon outran that of capture in more challenging samples. We found that capture was not as accurate as meta and amplicon in identifying between-sample variations, whereas amplicon method was not as accurate as the other two in investigating within-sample variations, suggesting amplicon sequencing was not suitable for studying virus-host interactions and viral transmission that heavily rely on intra-host dynamics. We illustrated that meta uncovered rich genetic information in the clinical samples besides SARS-CoV-2, providing references for clinical diagnostics and therapeutics. Taken all factors above and cost-effectiveness into consideration, we proposed guidance for how to choose sequencing strategy for SARSCoV-2 under different situations.

Conclusions: This is, to the best of our knowledge, the first work systematically investigating inter- and intraindividual variations of SARS-CoV-2 using amplicon- and capture-based whole-genome sequencing, as well as the first comparative study among multiple approaches. Our work offers practical solutions for genome sequencing and analyses of SARS-COV-2 and other emerging viruses.

Keywords: Emerging infectious diseases, COVID-19, Metatranscriptomic sequencing, Hybrid capture, Multiplex PCR, iSNV, Quasispecies, Genomic surveillance, Virus evolution

\section{Background}

As of 14 March 2020, severe acute respiratory syndrome coronavirus 2 (SARS-CoV-2) has surpassed severe acute respiratory syndrome coronavirus (SARS-CoV) and Middle East respiratory syndrome coronavirus (MERS-CoV) in every aspect, infecting over 140,000 people in more than 110 countries, with a mortality of over 5000 [1]. So far, coronaviruses have caused three major epidemics in the past two decades, posing a great challenge to global health and economy. Massively parallel sequencing (MPS) of viral genomes has demonstrated enormous capacity as a powerful tool to study emerging infectious diseases, such as SARS, MERS, Zika, and Ebola, in tracing the outbreak origin and drivers, tracking transmission chains, mapping the spread, and monitoring the evolution of the etiological agents [2-7]. Though by 14 March 2020, fewer than 500 SARS-CoV-2 genomes were published on public databases including China National GeneBank DataBase (CNGBdb), NCBI GenBank, and the Global Initiative on Sharing All Influenza Data (GISAID), and much remains unknown about the epidemiology and evolution of the virus. One possible explanation of the paucity of published SARS-CoV-2 genomes was the challenges posed by sequencing clinical samples with low virus abundance.

The first teams obtained the SARS-CoV-2 genome sequences through metatranscriptomic MPS, supplemented by PCR and Sanger sequencing of a combination of bronchoalveolar-lavage fluid (BALF) and culture [810] or from BALF directly [11]. Experience from studying SARS-CoV showed that BALF from the lower respiratory tract was an ideal sample type with higher viral load [12]. However, BALF was not routinely collected from every patient, and human airway epithelial (HAE) cell culture is very labor-intensive and time-consuming, taking 4 to 6 weeks $[9,13]$. Chan et al. managed to get the whole-genome sequences through metatranscriptomic sequencing with Oxford Nanopore platform supplemented by Sanger sequencing from both nasopharyngeal and sputum specimens after singleprimer amplification [14]. Holshue et al. published the whole-genome sequence using oropharyngeal and nasopharyngeal specimens through Sanger and metatranscriptomic sequencing with both Illumina and MinIon [15]. To date, multiplex PCR-based or hybrid capturebased whole-genome sequencing of SARS-CoV-2, as well as comparative studies between different approaches, have not been reported on peer-reviewed journals.

Besides inter-individual variations, dissecting intraindividual dynamics of viruses also largely promotes our understanding of virus-host interactions, viral evolution, and transmission as demonstrated for Ebola, Zika, Influenza, etc. [5, 16-18]. The analyses of intra-individual single nucleotide variations (iSNVs) and its allele frequency have also contributed to anti-viral therapy and drug resistance, e.g., to reveal highly conserved genes during the outbreak that potentially serve as ideal therapeutic targets $[17,19]$. However, it is challenging to accurately detect iSNVs from clinical samples, especially when the samples are subjected to extra steps of enrichment and amplification.

Therefore, we aim to comprehensively compare the sensitivity, inter-individual (variant) and intra-individual (iSNV) accuracy, and other general features of different approaches by systematically utilizing ultra-highthroughput metatranscriptomic, hybrid capture-based, and amplicon-based sequencing approaches to obtain genomic information of SARS-CoV-2 from serial 
dilutions of a cultured isolate and directly from clinical samples. We present a reasonable sequencing strategy that fits into different scenarios and estimate the minimal amount of sequencing data for downstream SARS-CoV-2 genome analyses. Our study offers practical solutions to facilitate the studies of SARS-CoV-2 and other emerging viruses in the future and would promote extensive genomic sequencing and analyses of SARS-CoV-2 and other emerging viruses, which would in turn contribute to real-time virus surveillance and managing viral outbreaks. Benefiting from our experimental workflows and bioinformatic pipelines, BGI Group has launched a Global Initiative on Open-source Genomics for SARS-CoV-2 (GIOG-S, https://giogs.genomics.cn/) and makes its platforms for multiplex PCR sequencing and ultra-deep metatranscriptomic sequencing available to global research teams within GIOG-S.

\section{Methods}

Sampling, RNA extraction, reverse transcription, and qRTPCR

Clinical specimens (including throat swab, nasal swab, anal swab, and sputum) were obtained from confirmed COVID-19 cases at the First Affiliated Hospital of Guangzhou Medical University. Total RNA of the cultured isolate of SARS-CoV-2 was obtained from the Academy of Military Medical Science (AMMS) and subjected to 10-fold serial dilutions. Virus isolation and RNA extraction were done in a biosafety level (BSL) 2+ laboratory with BSL-3 protection. Total RNA was extracted directly from the clinical specimens without inactivating the virus with QiAamp RNeasy Mini Kit (Qiagen, Heiden, Germany) following the manufacturer's instructions without modification. Real-time reverse transcription PCR (qRT-PCR) targeting RdRp gene and $\mathrm{N}$ gene of SARS-CoV-2 was used to detect and quantify the viral RNA within clinical samples and serial dilutions of the cultured isolate using the SARS-CoV-2 Nucleic Acid Detection Kit following the manufacture's protocol (Geneodx, Shanghai, China, and BGI-Shenzhen, Shenzhen, China).

\section{Metatranscriptomic library preparation and sequencing}

Host DNA was removed from RNA samples using DNase I, and the concentration of RNA samples was measured by Qubit RNA HS Assay Kit (Thermo Fisher Scientific, Waltham, MA, USA). DNA-depleted and purified RNA was used to construct the double-stranded (ds) circular DNA library with MGIEasy RNA Library preparation reagent set (MGI, Shenzhen, China), as follows: (1) RNA was fragmented by incubating with fragmentation buffer at $87^{\circ} \mathrm{C}$ for $6 \mathrm{~min}$; (2) ds cDNA was synthesized using random hexamers with fragmented RNA; (3) ds cDNA was subjected to end repair, adaptor ligation, and 18-cycle PCR amplification; and (4) PCR products were unique dual indexed (UDI), before going through circularization and rolling circle replication (RCR) to generate DNA nanoball (DNB)-based libraries. Negative controls prepared from nuclease-free water and total RNA isolated from human Michigan Cancer Foundation-7 (MCF-7) breast cancer cells were included. DNB preps of clinical samples were sequenced on the ultra-high-throughput DNBSEQ-T7 platform (MGI, Shenzhen, China) with paired-end $100 \mathrm{nt}$ strategy, generating $321 \mathrm{~Gb}$ sequencing data for each sample on average.

Outside of mainland China, BGI (https://www.bgi.com/ global/) has regional headquarters in Europe (Copenhagen, Denmark), Asia Pacific (Hong Kong, China), and Americas (San Jose, CA, USA) and has been actively serving customers in more than 66 countries. MGI (https://en.mgitech. $\mathrm{cn} /$ ) operates in 39 countries and regions with branches including Hong Kong in China, Kobe in Japan, Dubai in UAE, Riga in Latvia, and San Jose in the USA. The global training and service network is located in major countries and regions on 6 continents, with 40 training/after-sales service centers. To acquire reagents, instruments, and technical support, researchers may find regional contact from the official website or directly send enquiries to the specified corresponding author Y.Z. (zhangyanyan@genomics.cn).

\section{Hybrid capture-based enrichment and sequencing}

A hybrid capture technique was used to enrich SARS-CoV2-specific content from the metatranscriptomic doublestranded DNA libraries with the 2019-nCoVirus DNA/ RNA Capture Panel (BOKE, Beijing, China). Negative controls prepared from nuclease-free water and total RNA isolated from human MCF-7 breast cancer cells were included. The manufacturer's instructions were slightly modified to accommodate the MGISEQ-2000 platform, i.e., blocker oligos and PCR primer oligos were replaced by MGIEasy exon capture assistive kit (MGI, Shenzhen, China). DNB-based libraries were constructed and sequenced on the MGISEQ2000 platform with paired-end $100 \mathrm{nt}$ strategy using the same protocol described above, generating $37 \mathrm{~Gb}$ sequencing data for each sample on average.

\section{Amplicon-based enrichment and sequencing}

Total RNA was reverse transcribed to synthesize the first-strand cDNA with random hexamers and Superscript II reverse transcriptase kit (Invitrogen, Carlsbad, USA). Sequencing was attempted on all samples regardless of $\mathrm{Ct}$ value including negative controls prepared from nuclease-free water and NA12878 human gDNA. A two-step SARS-CoV-2 genome amplification was performed with an equimolar mixture of primers using ATOPlex SARS-CoV-2 Full Length Genome Panel following the manufacture's protocol (MGI, Shenzhen, China), generating $137 \times \sim 400$ bp amplicons or $299 \times \sim$ 
$200 \mathrm{bp}$ amplicons, and the genome positions of the amplicons are shown in Additional file 1 Table S1. Twenty microliters of first-strand CDNA was mixed with the components of the first PCR reaction following the manufacturer's instructions. Two nanograms of human gDNA was added to each PCR reaction of the cultured isolate. A set of controls was adopted to help quantifying viral load and identify potential contamination. During library construction for amplicon sequencing, each sample was mixed with a fixed copy number of lambda genomic DNA (external control), and the external control and the SARS-CoV-2 genomes were amplified at the same time. The PCR was performed as follows: $5 \mathrm{~min}$ at $37^{\circ} \mathrm{C} ; 10 \mathrm{~min}$ at $95^{\circ} \mathrm{C} ; 15$ cycles of $10 \mathrm{~s}$ at $95^{\circ} \mathrm{C}, 1 \mathrm{~min}$ at $64^{\circ} \mathrm{C}, 1 \mathrm{~min}$ at $60^{\circ} \mathrm{C}$, to $10 \mathrm{~s}$ at $72^{\circ} \mathrm{C}$; and $2 \mathrm{~min}$ at $72{ }^{\circ} \mathrm{C}$. The products were purified with MGI EasyDNA Clean beads (MGI, BGI-Shenzhen, China) at a 5:4 ratio and cleaned with $80 \%$ concentration ethanol according to the manufacturer's instructions. The 2nd PCR was performed under the same regimen as the 1st PCR except for 25 cycles, and the bead-purified products from the first PCR reaction were unique dual indexed. After the 2nd PCR, products were purified following the same procedures as the 1st PCR and quantified using the Qubit dsDNA High Sensitivity assay on Qubit 3.0 (Life Technologies). PCR products of samples yielding sufficient material $(>5 \mathrm{ng} / \mu \mathrm{l})$ were pooled at equimolar to a total DNA amount of $300 \mathrm{ng}$ before converting to single-stranded circular DNA. DNB-based libraries were generated from $20 \mu \mathrm{l}$ of single-stranded circular DNA pools and sequenced on the MGISEQ-2000 platform with single-end $400 \mathrm{nt}$ strategy, generating $1.8 \mathrm{~Gb}$ sequencing data for each sample on average.

\section{Identification of Coronaviridae-like reads in massively parallel sequencing data}

For metatranscriptomic and hybrid capture sequencing data, total reads were first processed using Kraken v0.10.5 [20] (default parameters) with a self-built database of Coronaviridae genomes (including SARS, MERS, and SARS-CoV-2 genome sequences downloaded from GISAID, NCBI, and CNGB) to identify Coronaviridaelike reads in a loose manner. fastp v0.19.5 [21] (parameters: -q 20 -u 20 -n $1-1$ 50) and SOAPnuke v1.5.6 [22] (parameters: -1 20 -q 0.2 -E 50 -n 0.02 -5 0 -Q 2 -G -d) were used to remove low-quality reads, duplications, and adaptor contaminations. Low-complexity reads were then removed using PRINSEQ v0.20.4 [23] (parameters: -lc_method dust -lc_threshold 7). Samples that exhibited higher coverage and at least 10-fold higher average sequencing depth than negative controls were accepted for downstream analyses of inter- and intra-individual variations.
For amplicon sequencing data, SE400 reads were first processed with fastp v0.19.5 [21] (parameters: -q $20-\mathrm{u}$ 20 -n 1 -1 50) to remove low-quality reads and adaptor sequences. Primer sequences and the $21 \mathrm{nt}$ upstream and downstream of primers within the reads were then trimmed with BAMClipper v1.1.1 [24] (parameters: -n 4 -u 21 -d 21). Reads with low-quality bases, adaptors, primers, and adjacent sequences completely removed as described above were considered as clean reads for downstream analyses. The viral load of SARS-CoV-2 was quantified based on the data from both external control and the target virus. We define a $C=$ target viral load/(target viral load + external control load), and a $C>$ $0.1 \%$ of negative groups (prepared from human nucleic acids and nuclease-free water) indicates unacceptable contamination. Further, we consider a sample acceptable only when the $C$ value of the sample is an order of magnitude higher than that of negative groups, for instance, if $C<0.01 \%$ for all negative controls and $C>0.1 \%$ for an experimental group.

\section{Genome assembly of SARS-CoV-2}

For metatranscriptomic and hybrid capture sequencing, the Coronaviridae-like reads of samples with $<100 \times$ average sequencing depth were directly de novo assembled with SPAdes (v3.14.0, [25]) using the default settings. The Coronaviridae-like reads of samples with > $100 \times$ average sequencing depth across SARS-CoV-2 genome were subsampled to achieve $100 \times$ sequencing depth before being assembled.

For amplicon sequencing, SARS-CoV-2 consensus sequences were generated using Pilon v1.23 [26] (parameters: --changes -vcf --changes --vcf --mindepth 1 --fix all, amb). Nucleotide positions with sequencing depth < $100 \times$ or $<5$-fold higher than that of negative controls were masked as ambiguous base $\mathrm{N}$.

\section{Visualization of coverage depth across the viral genomes} The Coronaviridae-like reads from metatranscriptomic and hybrid capture sequencing data were aligned against the SARS-CoV-2 reference genome (GISAID accession: EPI_ISL_402119) [27] with BWA aln (v0.7.16) [28]. Duplications were identified by Picard Markduplicates (v2.10.10) (http://broadinstitute.github.io/picard) with default settings. For each sample, we calculated the depth of coverage at each nucleotide position of the SARS-CoV-2 reference genome with SAMtools (v1.9) [29] and scaled the values to the mean depth. For each nucleotide position, we calculated the median depth and 20th and 80th percentiles across all samples. Coverage depth across the SARS-CoV-2 reference genome was plotted within a 200-nt sliding window using the ggplot2 [30] package in $\mathrm{R}$ (v3.6.1) [31]. 
Amplicon sequencing data was processed as described above, except that duplications were not removed. A heatmap was generated to visualize the viral genome coverage for all samples sequenced by the amplicon method with the pheatmap v1.0.12 [32] package in $R$ (v3.6.1) [31]. The depth at each nucleotide position was binarized and was shown in blue if depth of coverage was $100 \times$ and above.

\section{Relationship between viral load and minimum sequencing output across methods}

SARS-CoV-2 reads of metatranscriptomic and hybrid capture sequencing data were identified by aligning the Coronaviridae-like reads against the SARS-CoV-2 reference genome (GISAID accession: EPI_ISL_402119) [27] with BWA in a strict manner of coverage $\geq 95 \%$ and identity $\geq 90 \%$. For comparisons of the coverage depth of the viral genome across samples and methods, we normalized the viral reads to total sequencing reads with SARS-CoV-2 reads per million (SARS-CoV-2-RPM). SARS-CoV-2-RPM for amplicon sequencing data was calculated by the same pipeline applied for metatranscriptomic and hybrid capture sequencing data.

To estimate the minimum data required for genome assembly and genome variation analysis, we applied gradient-based sampling to the SARS-CoV-2 genome alignments (referred to BAM files) to each dataset using SAMtools (v1.9) [29]. The effective genome coverage was set as $95 \%$ for all three MPS methods. Considering the distinct technologies used in different methods, we set method-dependent thresholds of effective depth as follows: (1) $\geq 10 \times$ for metatranscriptomic sequencing, (2) $\geq 20 \times$ for hybrid capture sequencing, and ( 3$) \geq 100 \times$ for amplicon sequencing. We next calculated the coverage and depth within each subsampled BAM file per sample to determine the minimal BAM file that could meet the above thresholds of both coverage and sequencing depth. The method-dependent minimum sequencing output of each sample was estimated accordingly. We assessed the correlations between the SARS-CoV-2 genome copies per milliliter in diluted samples of cultured isolates and the minimum sequencing output for amplicon- and capture-based methods using Pearson's correlation coefficient $(R)$ with the function scatter from the $\mathrm{R}$ package (v3.6.1) ggpubr (v0.2.5) [33].

\section{Analysis of inter- and intra-individual variants}

For metatranscriptomic and hybrid capture sequencing data, variant calling was performed based on the BAM files of identified SARS-CoV-2 reads after removing duplications using Picard Markduplicates (http://broadinstitute.github.io/picard). Amplicon sequencing data were processed as described above, except that duplications were not removed. Variants were first called with freebayes (v1.3.1) [34] (parameters: -p 1 -q 20 -m 60 --min-coverage $10-\mathrm{V}$ ), and the low-confidence variants were removed with snippy-vcf_filter (v3.2) [35] (parameters: --minqual 100 --mincov 10 --minfrac 0.8$)$. The remaining variants in VCF files generated by freebayes were annotated in SARS-CoV-2 genome assemblies and consensus sequences with SNVeff (v4.3) [36] using default parameters. Next, pysamstats v1.1.2 (https:/github. com/alimanfoo/pysamstats) (parameters: -type variation strand --min-baseq 20 -D 1000000) was used to count the number of matches, mismatches, deletions, and insertions at each base to determine the allele frequencies. Variant calls with allele frequencies $\geq 80 \%$ were identified as SNVs.

Nucleotide positions with $\geq 100 \times$ sequencing depth from amplicon sequencing, $\geq 10 \times$ from metatranscriptomic sequencing, and $\geq 20 \times$ from capture sequencing were kept for iSNV calling. The candidate iSNVs were further filtered as follows: (1) frequency filtering, only minor alleles (frequency $\geq 5 \%$ and $<50 \%$ ) and major alleles (frequency $\geq 50 \%$ and $\leq 95 \%$ ) were remained; (2) depth filtering, iSNVs with fewer than five forward or reverse reads were removed; and (3) strand bias filtering (not applicable to single-end reads of amplicon sequencing), iSNVs were removed if there were more than a 10 -fold strand bias or a 5-fold difference between the strand bias of the variant call and that of the reference call.

\section{Taxonomy of clinical samples by unbiased metatranscriptomic sequencing}

For metatranscriptomic sequencing of clinical samples, raw sequencing data of a single sequence lane (approximately 60-75 Gb per sample) was used to simultaneously assess the RNA expression patterns of human, bacteria, and viruses in clinical samples from COVID-19 patients. We first used the software fastp (v0.19.5) [21] to filter low-quality reads and remove adapter with parameters: -5 -3 -q 20 -c -1 30. After QC, we mapped high-quality reads to hg19 and removed human ribosomal RNA (rRNA) reads by using SOAP2 v2.21 [37] (parameters: -m 0 -x 1000 -s 28 -1 32 -v 5 -r 1), and the remaining RNA reads were then aligned to hg19 by HISAT2 [38] with default settings to identify non-rRNA human transcripts as previously described. Next, we applied Kraken 2 [39] (version 2.0.8-beta, parameters: --threads 24 --confidence 0 ) to assign microbial taxonomic ranks to non-human RNA reads against the large reference database MiniKraken2 (April 2019, 8 GB) built from the Refseq bacteria, archaea, and viral libraries and the h38 human genome. Bracken [40] (Bayesian Reestimation of Abundance with Kraken) was further applied to estimate microbial relative abundances based on taxonomic ranks of reads assigned by Kraken2. 


\section{Results}

\section{Design of the comparative study}

We sampled eight specimens from COVID-19 patients in February 2020, including throat swab, nasal swab, anal swab, and sputum specimens, and the corresponding cycle threshold (Ct) value of SARS-CoV-2 qRT-PCR ranges from 18 to 32 (Table 1). We initially tried to boost the coverage and depth of the viral genome by ultra-deep metatranscriptomic sequencing with an average sequencing output of 1,607,264,105 paired-end reads (Table 1). Although we managed to obtain complete viral genome assemblies for each specimen, the sequencing depth varied across specimens. Only $0.002-0.003 \%$ of the total reads were assigned to the SARS-CoV-2 in three samples (GZMU0014, GZM U0030, and GZMU0031) with Ct between 29 and 32, resulting in inferior sequencing depth (less than 100x) (Table 1). Isolating viruses and enriching them in cell culture might improve the situation, but this requires high-standard laboratory settings and expertise apart from being time-consuming. Also, unexpected mutations that are not concordant with original clinical samples may be introduced during the culturing process.

To enrich adequate viral content for whole-genome sequencing in a convenient manner, we pursued two other methods: multiplex PCR amplification (amplicon) and hybrid capture (capture) (Fig. 1). We designed a systematic study to comprehensively validate the uniformity, sensitivity, and inter-individual (variant) and intraindividual (iSNV) accuracy of multiple approaches by sequencing serial dilutions of a cultured isolate (unpublished), as well as the eight clinical samples (Fig. 2). We performed qRT-PCR of 10-fold serial dilutions (D1-D7) of the cultured isolate, and the $\mathrm{Ct}$ was 17.3, 20.8, and 24.5 for $28.7,31.8,35$, and 39.9, respectively, indicating the undiluted RNA (D0) of the cultured isolate contained $\sim 1 \mathrm{E}+08$ genome copies per milliliter. For amplicon sequencing, we utilized two kits comprising two sets of primers generating PCR products of $300-400 \mathrm{bp}$ and $100-200 \mathrm{bp}$, respectively. The $\sim 400 \mathrm{bp}$ amplicon-based sequencing was implemented in all samples and analyzed throughout the study, while the $\sim 200 \mathrm{bp}$ amplicon-based sequencing was only applied in the cultured isolate for coverage analysis.

\section{Comparison of uniformity and sensitivity}

Theoretically, amplicon sequencing should be the most sensitive and economical method among the three and is particularly suitable in an outbreak where viral isolates are highly related. Although, there are still potential pitfalls, for instance, the 40-cycle PCR in our workflow might augment trace amounts of SARS-CoV-2 crosscontamination. To ensure the confidence of the datasets, we included serial dilutions of the cultured isolate and negative controls prepared from nuclease-free water and human nucleic acids since the 1st PCR. All samples in $400 \mathrm{bp}$ amplicon-based sequencing exhibited >99.5\% coverage of $1 \times$ depth across the SARS-CoV-2 genome except for 1E+01 (95.23\%), GZMU0031 (73.65\%), HNA $(6.17 \%)$, and water $(60.24 \%)$ and $>97.00 \%$ coverage of $100 \times$ depth across the SARS-CoV-2 genome except for GZMU0030 (94.15\%), GZMU0042 (88.17\%), GZMU0014 (71.66\%), D7 (39.49\%), GZMU0031 (0.00\%), HNA $(0.00 \%)$, and water $(0.00 \%)$, suggesting the primers were well designed and the positive datasets were reliable (Fig. 3a). We also set stringent and method-specific criteria to filter low-confidence sequencing reads and samples based on a set of controls (see the "Methods" section). Another pitfall is that amplification across the genome can hardly be unbiased, causing difficulties in complete genome assembly. Indeed, amplicon sequencing exhibited a lower level of uniformity compared with metatranscriptomic sequencing, in terms of coverage across the viral genomes from the cultural isolate and the clinical samples tested in our study (Fig. 3b, d; Additional file 2 Fig. S1). To our surprise, however, capture

Table 1 Metatranscriptomic sequencing data summary of eight SARS-CoV-2-positive clinical samples collected from Guangzhou in February 2020

\begin{tabular}{|c|c|c|c|c|c|c|c|}
\hline Sample ID & $\begin{array}{l}\text { Sample } \\
\text { type }\end{array}$ & $\mathrm{Ct}$ & $\begin{array}{l}\text { No. of sequencing read } \\
\text { pairs }\end{array}$ & $\begin{array}{l}\text { No. of SARS-CoV-2 read } \\
\text { pairs }\end{array}$ & $\begin{array}{l}\text { Percentage of SARS-CoV-2 read } \\
\text { pairs }\end{array}$ & $\begin{array}{l}\text { Coverage } \\
(\%)\end{array}$ & $\begin{array}{l}\text { Depth } \\
(\times)\end{array}$ \\
\hline GZMU0047 & Nasal swab & 18 & $1,547,648,648$ & $85,316,930$ & 5.513 & 100 & 113,021 \\
\hline GZMU0016 & Sputum & 21 & $1,578,573,142$ & $7,489,563$ & 0.474 & 99.96 & 12,734 \\
\hline GZMU0048 & $\begin{array}{l}\text { Throat } \\
\text { swab }\end{array}$ & 24 & $1,647,198,588$ & $3,365,330$ & 0.204 & 99.91 & 6508 \\
\hline GZMU0044 & Nasal swab & 26 & $1,609,367,415$ & $7,275,402$ & 0.452 & 99.92 & 12,758 \\
\hline GZMU0030 & $\begin{array}{l}\text { Throat } \\
\text { swab }\end{array}$ & 29 & $1,725,727,056$ & 31,148 & 0.002 & 99.87 & 69 \\
\hline GZMU0014 & Sputum & 30 & $1,596,713,550$ & 46,199 & 0.003 & 99.9 & 95 \\
\hline GZMU0042 & Sputum & 32 & $1,481,162,934$ & 567,266 & 0.038 & 99.94 & 1133 \\
\hline GZMU0031 & Anal swab & 32 & $1,671,721,507$ & 25,392 & 0.002 & 99.89 & 14 \\
\hline
\end{tabular}




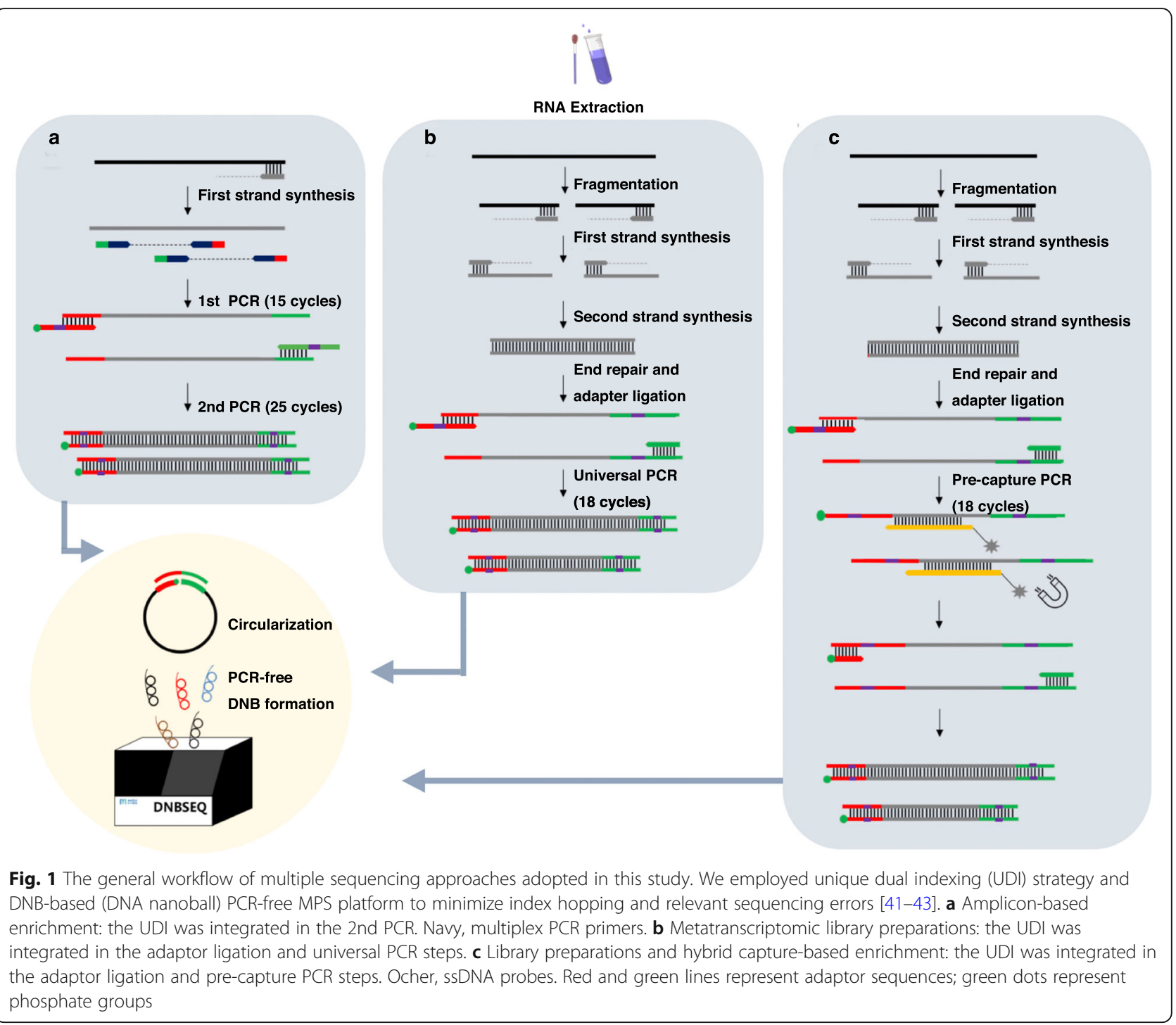

sequencing was almost as uniform as meta sequencing, demonstrating better performance than the previous capture method used to enrich ZIKV despite that SARSCoV-2 genome is $\sim 3$-fold larger than ZIKV [44] (Fig. 3b, c). Two reasons among others were likely to be accountable to this improvement: (1) we utilized 506 pieces of 120 ssDNA probes covering $2 \times$ of the SARS-CoV- 2 genome to capture the libraries, and (2) we employed the DNBSEQ sequencing technology that features PCR-free rolling circle replication (RCR) of DNA nanoballs (DNBs) [41, 42].

The sequencing results of amplicon and capture approaches revealed dramatic increases in the ratio of SARS-CoV-2 reads out of the total reads compared with meta sequencing, suggesting the enrichment was highly efficient-5596-fold in capture method and 5710-fold in amplicon method for each sample on average (Additional file 2 Table S2-S3). To further compare the sensitivity of different methods, we plotted the number of SARS-CoV-2 reads per million (SARS-CoV-2-RPM) of total sequencing reads against the viral concentration for each sample. Meta sequencing produced significantly lower SARS-CoV-2-RPM than the other two methods among clinical samples tested with a wide range of $\mathrm{Ct}$ values (Fig. 3e). The productivity was similar between the other two methods when the input RNA of the cultured isolate contained $1 \mathrm{E}+05$ genome copies per milliliter and above (Fig. 3e). However, amplicon sequencing produced 10 - to 100 -fold more SARS-CoV- 2 reads than capture sequencing when the input RNA concentration of the cultured isolate was $1 \mathrm{E}+04$ genome copies per milliliter and lower, suggesting amplicon-based enrichment was more efficient than capture for more challenging samples (conc. $\leq 1 \mathrm{E}+04$ genome copies per milliliter, or $\mathrm{Ct} \geq 28.7$ ) (Fig. 3e). Meta sequencing-as expected-produced dramatically lower SARS-CoV-2-RPM 


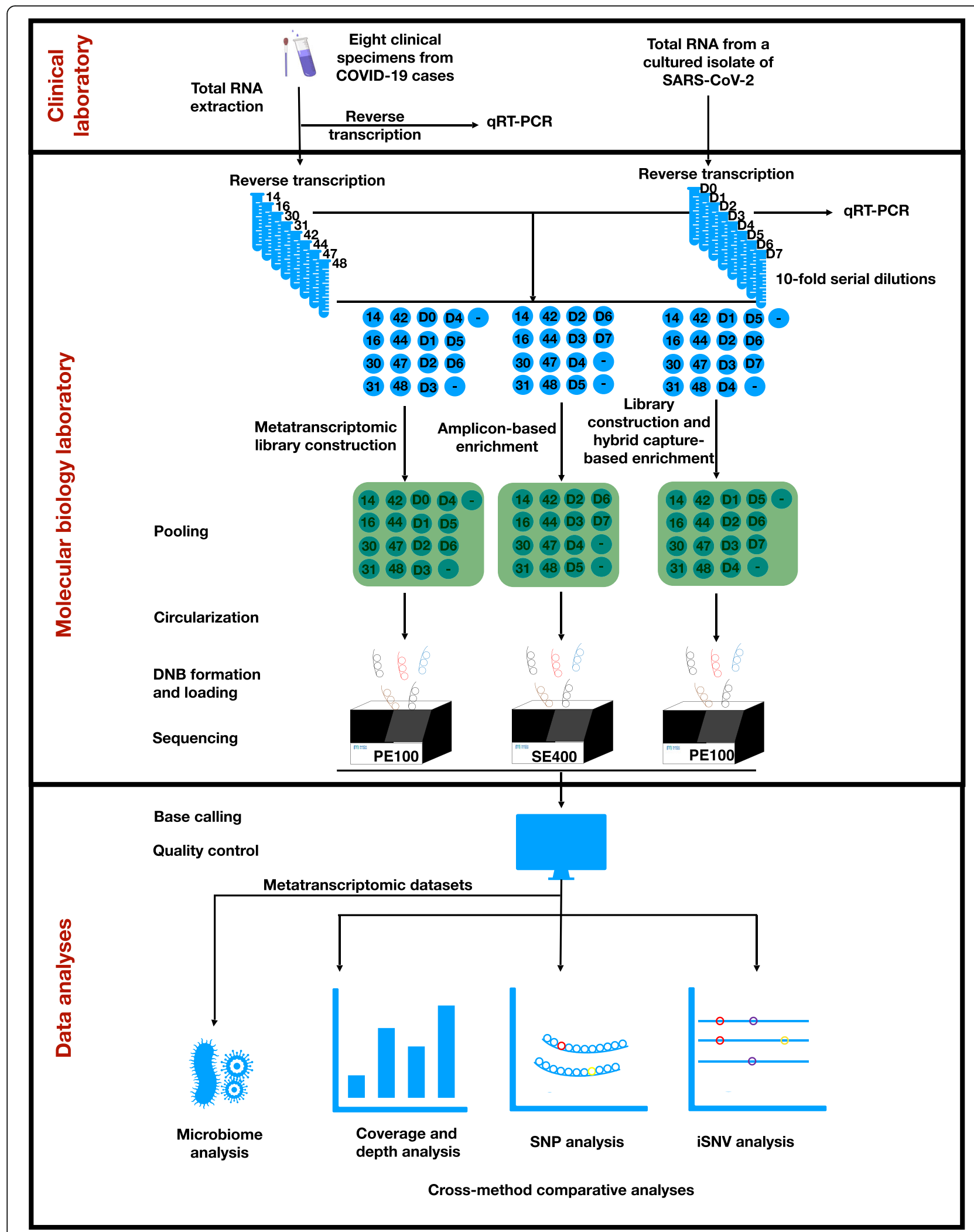

Fig. 2 (See legend on next page.) 
(See figure on previous page.)

Fig. 2 Overview of the study design. Eight clinical samples and serial dilutions of a cultured isolate were subjected to direct metatranscriptomic library construction, amplicon-based enrichment, and hybrid capture-based enrichment, respectively. Libraries generated from each method were pooled, respectively. DNB, DNA nanoball. 14, GZMU0014; 16, GZMU0016; 30, GZMU0030; 31, GZMU0031; 42, GZMU0042; 44, GZMU0044; 47, GZMU0047; 48, GZMU0048. D0, undiluted sample of the cultured isolate; D1-D7, seven serial diluted samples of the cultured isolate, ranging from $1 \mathrm{E}+07$ to $1 \mathrm{E}+01$ genome copies per milliliter, in 10-fold dilution. "-", negative controls prepared from nuclease-free water and human nucleic acids. PE100, paired-end 100-nt reads; SE400, single-end 100-nt reads

than the other two methods among clinical samples tested with a wide range of $\mathrm{Ct}$ values, whereas amplicon and capture were generally comparable to each other (Fig. 3f). Considering the costs for sequencing, storage, and analysis increase substantially with larger datasets, we tried to estimate how much sequencing data must be produced for each approach in order to achieve $10 \times$ depth across $95 \%$ of the SARS-CoV-2 genome, and the results can be found in Additional file 2 Table 3. As a practical, cost-effective guidance for future sequencing, we also assessed the minimum sequencing output required to pass the stringent filters ( $\geq 95 \%$ coverage and method-specific depth, see the "Methods" section) in our pipelines corresponding to different viral loads. We estimated that for high-confidence downstream analyses, amplicon sequencing requires at least 2757 to 186 megabases $(\mathrm{Mb})$ for samples containing $1 \mathrm{E}+02$ to $1 \mathrm{E}+06$ copies of SARS-CoV-2 genome per milliliter, while capture sequencing requires 24,474 to $9 \mathrm{Mb}$ for the same situation (Fig. 3g, Additional file 2 Table S4-S5).

\section{Investigation of inter- and intra-individual variations}

To determine the accuracy of different approaches in discovering inter-individual genetic diversity, we tested each method in calling the single nucleotide variations (SNVs) and verified some of the SNVs with Sanger sequencing (Additional file 2 Fig. S2). Two to five SNVs were identified within each clinical sample, and in all the seven samples, SNVs identified by the three methods were concordant except that capture missed one SNV at position 16535 in GZMU0014 (Fig. 4a). We then investigated the allele frequencies of these sites across methods and found that alleles identified by capture sequencing displayed lower frequencies than the other two methods, especially for GZMU0014, GZMU0030, and GZMU0042 where the viral load was lower $(\mathrm{Ct} \geq 29)$, which explained why capture sequencing neglected an SNV in our pipeline when the cutoff of SNV calling was set as $80 \%$ allele frequency (Fig. 4b). These data indicate that amplicon sequencing is more accurate than capture sequencing in identifying SNVs, especially for challenging samples.

To further determine the accuracy of different approaches in identifying SARS-CoV-2 iSNVs, we examined minor allele frequencies in serial dilutions of the cultured SARS-CoV-2 isolate and clinical samples. For serial dilutions of the cultured isolate, the minor allele frequencies detected in capture sequencing datasets were generally approximate to meta sequencing, while most allele frequencies in amplicon sequencing datasets deviated with those in meta sequencing (Fig. 4c). A similar pattern was shown for clinical samples, indicating that amplicon sequencing was unreliable of quantifying minor allele frequencies (Fig. 4d). Plotting allele frequencies against SARS-CoV-2 concentrations supported the above finding and further revealed that amplicon sequencing was unreliable of allele frequencies at all concentrations while capture sequencing was reliable at $>1 \mathrm{E}+03$ genome copies per milliliter (Additional file 2 Fig. S3). Referring to the iSNV identified in clinical samples by meta sequencing, we then calculated the false positive rate (FPR) of minor alleles called by amplicon and capture methods. The FPR of minor alleles identified in amplicon sequencing was $0.74 \%$, while that in capture sequencing was $0.02 \%$. Together, these results suggest amplicon sequencing was not as accurate as capture sequencing in identifying minor alleles.

\section{Microbiome in clinical samples}

In addition to target viral genome, metatranscriptomic sequencing has also allowed us to investigate RNA expression patterns of the overall microbiome and host content and thus suitable for discovering new viruses, distinguishing co-infections, and dissecting virus-host interactions. To explore the microbiota, we performed further metatranscriptomic analysis of the clinical samples. We were able to identify host nucleic acids in all of the samples, and over $95 \%$ of total reads were from the host in sputum, nasal, and throat samples (Additional file 2 Fig. S4a). Virus contributed to less than $5 \%$ of reads in anal swab and throat swab while more than $50 \%$ of reads in nasal swab (Additional file 2 Fig. S4b). These results suggest nasal swab could be the most ideal sample type for viral detection among the four sample types, which agrees with recent clinical evidence [45]. Previous studies have compared different sample types of other coronaviruses using qRT-PCR and found that nasopharyngeal aspirates and throat and nose swabs appear to be the most useful clinical specimens in the first 5 days of illness caused by SARS-CoV infection [46] and nasal swabs are the candidate sample of choice for detecting MERS-CoV using qRT-PCR technology in apparently healthy camels [47]. 


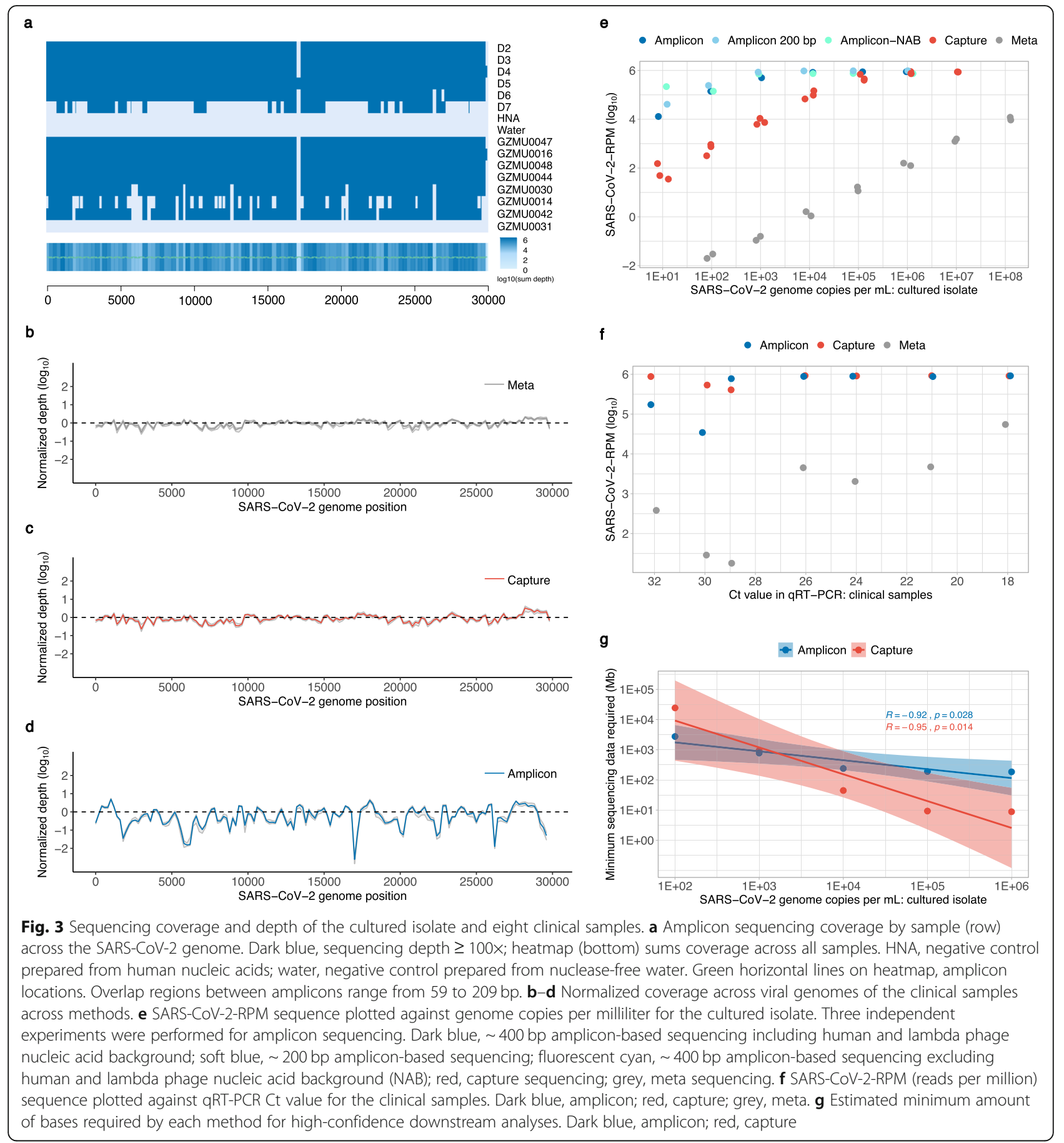

Among the viral reads, over $90 \%$ were Coronaviridae, which is consistent with clinical diagnostics (Additional file 2 Fig. S4c). Reads from other viruses were also identified, indicating further measurements could be taken to confirm if co-infection exists (Additional file 2 Fig. S4). Bacterial composition was also shown, providing support for scientific research, as well as for further confirmation of bacterial infection and antibiotics prescription (Additional file 2 Fig. S4d-f).

\section{Guidance for virus sequencing}

Taken together, each sequencing scheme elaborated here for massively parallel sequencing of SARS-CoV-2 genomes has its own merits (Table 2). We hereby propose a reasonable, cost-effective strategy for sequencing and analyzing SARS-CoV-2 under different situations: (1) if one wants to study other genetic materials than the target viruses, or the viruses become highly diversified via recombinational events, or the viral load within the 


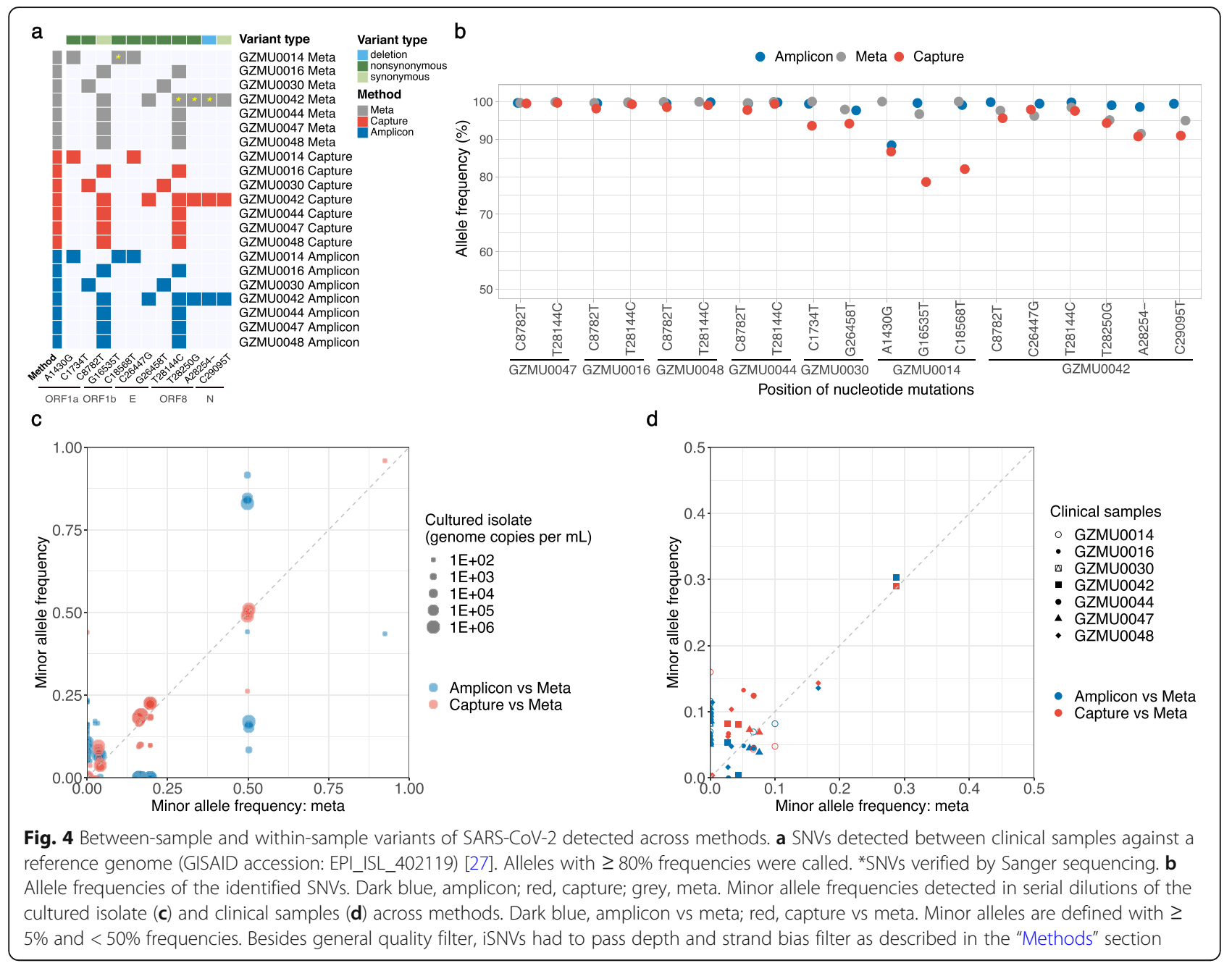

Table 2 General characteristics of the three approaches employed in this study

\begin{tabular}{|c|c|c|c|}
\hline & $\begin{array}{l}\text { Metatranscriptomic } \\
\text { sequencing }\end{array}$ & $\begin{array}{l}\text { Hybrid capture-based } \\
\text { sequencing }\end{array}$ & $\begin{array}{l}\text { Multiplex PCR amplicon-based } \\
\text { sequencing }\end{array}$ \\
\hline Sequencing objective & Microbiome + human & Target genome & Target genome \\
\hline 2nd strand synthesis & Y & Y & N \\
\hline Fragmentation & Y & Y & N \\
\hline Library preparation & Y & Y & N \\
\hline PCR & 18 cycles & $18+18$ cycles & $15+25$ cycles \\
\hline Estimated time for library construction & $10.5 \mathrm{~h}$ & $20.5 \mathrm{~h}$ & $7.5 \mathrm{~h}$ \\
\hline Oligo synthesis & - & $120 \mathrm{nt} \times 506$ & $40-60 \mathrm{nt} \times 2 \times(113+14+10)$ \\
\hline Estimated cost per sample (USD) ${ }^{a}$ & 112.86 & 65.14 & 48.00 \\
\hline $\begin{array}{l}\text { Estimated minimum data for downstream analyses } \\
\text { (base level) }\end{array}$ & $>10 \mathrm{~Gb}$ & $\mathrm{Mb}$ & $\mathrm{Mb}$ \\
\hline Uniformity & High & Moderate & Low \\
\hline Sensitivity & + & ++ & +++ \\
\hline Accuracy (SNV) & +++ & ++ & +++ \\
\hline Accuracy (iSNV) & +++ & ++ & + \\
\hline
\end{tabular}

${ }^{\mathrm{a}}$ The price varies greatly with different sequencing output and in different regions 
RNA sample is high (e.g., conc. $\geq 1 \mathrm{E}+05$ viral genome copies per milliliter, or $\mathrm{Ct} \leq 24.5$ ), meta sequencing can be prioritized; (2) if one focuses on intra-individual variations for more challenging samples (e.g., conc. $<1 \mathrm{E}+05$ and $>1 \mathrm{E}+03$ viral genome copies per milliliter, or $\mathrm{Ct}>$ 24.5 and $<31.8$ ), capture sequencing seems to be a justified choice; and (3) if identifying SNVs is the main purpose, the most convenient, economical strategy would be amplicon sequencing that can support analyses of samples containing lower than $1 \mathrm{E}+05$ viral genome copies per milliliter, or $\mathrm{Ct}>24.5$.

\section{Discussion}

Sequencing low-titer viruses directly from clinical samples is challenging, especially for coronaviruses that are the largest among RNA viruses ( 3-fold larger compared with ZIKV). Isolating viruses and enriching them in cell culture require high-standard laboratory settings and expertise apart from being time-consuming. The enrichment methods presented here have several advantages-to different degrees-over the other existing protocols $[48,49]$. Firstly, the multiplex PCR protocol for ZIKV sequencing [48] and the Artic Network protocol for SARS-CoV-2 sequencing [49] require library preparation after PCR. Our amplicon method is more convenient since the barcoding and adaptor ligation steps are integrated into the PCR process; in other words, the PCR products are the library. Secondly, we adopt a set of controls to help us quantify viral load and identify potential contamination. During library construction for amplicon sequencing, each sample was mixed with standard lambda genomic DNA (external control), and the external control and the SARS-CoV-2 genomes are amplified at the same time. After sequencing, the viral load of SARS-CoV-2 is quantified based on the data from both external control and the target virus. We define a $C=$ target viral load/(target viral load + external control load), and a $C>0.1 \%$ of negative groups (prepared from human nucleic acids and nuclease-free water) indicates severe contamination. Further, we consider a sample acceptable only when the $C$ value of the sample is an order of magnitude higher than that of negative groups, for instance, if $C<0.01 \%$ for all negative controls and $C>0.1 \%$ for an experimental group. Finally, our work is the first that focuses around the use of BGI and MGI materials and platforms while previous protocols were mainly designed for Illumina or Oxford Nanopore Technologies (ONT).

Compared with direct metatranscriptomic sequencing, hybrid capture and amplicon sequencing methods are more sensitive but less accurate and neither of the two can be used to sequence highly diverse or recombinant viruses because the primers and probes are specific to known viral genomes. Although amplicon sequencing compromises its accuracy, it becomes the most convenient and economical method of all. Either or a combination of the approaches described here can be chosen to cope with various needs of researchers, e.g., metatranscriptomic sequencing data with insufficient coverage and depth can be pooled with hybrid capture data to generate high-quality assemblies [44]. From the perspective of virologists who conduct genomic studies of SARS-CoV-2, one dilemma is that there is hardly any standard of which sequencing method should be chosen for different samples or research purposes. Clinical specimens are precious, and it is unlikely to test each method on them. Of course, time and cost are also important factors that are needed to be considered. Therefore, in this work, we systematically examined the advantages and disadvantages of each method using different samples and proposed a guidance for rationally choosing the most suitable approach.

Moreover, we estimated the minimum sequencing output required for different samples using each method. This is another frequently encountered question, because larger output requires higher sequencing expenses, larger storage space, and more computing resources. The most cost-effective way is to generate the minimum amount of data needed for downstream analyses. Our work provides practical help for researchers to estimate how much data is necessary, although it varies with experimental procedures (e.g., total RNA extraction, rRNA depletion) and sample types (e.g., nasal swab typically requires less data than other sample types), and thus should be determined case by case.

Some advantages and disadvantages described above might be specific to the experimental workflows and bioinformatic pipelines presented in the current work, for instance, (1) the uniformity of amplicon sequencing can be improved by reducing the amount of cycles in the 1st PCR to 13 while increasing that in the 2nd PCR to 17 or increasing the molar ratios of primers targeting the region with low coverage, e.g., genomic position 1696517246; (2) the amplicon sequencing is particularly convenient compared with previous counterparts since the standard fragmentation and library construction steps are omitted here by integrating adaptor and barcode ligation in the 2nd PCR and sequencing the amplicons using single-end $400 \mathrm{nt}$ reads; (3) using less than 506 pieces of 120 ssDNA probes in hybrid capture may attenuate the sequencing coverage while decrease the uniformity; (4) metatranscriptomic sequencing was conducted with an ultra-high-throughput sequencing platform so that the successful rate was substantially higher than usual; and (5) the minimal amount of data necessary for analyzing the SARS-CoV-2 genome from clinical samples across methods can be deviated from that predicted by data from the cultured isolate, and this was 
possibly due to the fact that nucleic acid background from the host and other microbes varies (Additional file 2 Table S4-S5, Additional file 2 Fig. S4). Also, we do not consider the time spent in sequencing since the workflows can be easily adapted to various platforms including Illumina and ONT, besides DNBSEQ of MGI.

\section{Conclusions}

All three methods can effectively obtain SARS-CoV-2 genome information from clinical samples and can be used to study genome variations of the virus. However, the sensitivity, accuracy, and cost of the three methods vary greatly, and thus, each method must be rationally chosen to cope with different research purposes and different clinical samples. This work offers practical guidance for genome sequencing and analyses of SARS$\mathrm{CoV}-2$ and other emerging viruses.

\section{Supplementary information}

Supplementary information accompanies this paper at https://doi.org/10. 1186/s13073-020-00751-4.

Additional file 1: Table S1. Genome positions of amplicons.

Additional file 2: Fig. S1. Normalized coverage across SARS-CoV-2 genomes of serial dilutions of the cultured isolate sequenced by multiple approaches. Fig. S2. Sanger sequencing results of SNVs in clinical samples. Fig. S3. Box-plot of allele frequencies against SARS-CoV-2 genome copies per mL. Fig. S4. Taxonomy of clinical samples by unbiased metatranscriptomic sequencing. Table S2. Capture sequencing data summary of serial dilutions of the cultured isolate and eight SARS-CoV-2 positive clinical samples collected from Guangzhou (PE100). Table S3. Amplicon sequencing data summary of serial dilutions of the cultured isolate and eight SARS-CoV-2 positive clinical samples collected from Guangzhou (SE400). Table S4. Estimated minimum amount of sequencing data required to achieve $\geq 10 X$ sequencing depth and $\geq 95 \%$ coverage for different methods. Table S5. Estimated minimum amount of sequencing data required to achieve high-confidence variants calling analyses for different methods. Table S6. Bioinformatic tools and parameters used in this study.

Additional file 3: Table S7. Genome sequences acknowledged.

\section{Abbreviations \\ MPS: Massively parallel sequencing; COVID-19: Coronavirus disease 2019; SARS-CoV: Severe acute respiratory syndrome coronavirus; MERS-CoV: Middle East respiratory syndrome coronavirus; BALF: Bronchoalveolar-lavage fluid; HAE: Human airway epithelial; iSNV: Intra-individual single nucleotide variation; Ct: Cycle threshold; RCR: Rolling circle replication; DNB: DNA nanoball; RPM: Reads per million; UDI: Unique dual indexing; HNA: Human nucleic acid; NAB: Nucleic acid background}

\section{Acknowledgements}

We attribute this work to the amazing people in this land who dedicate themselves to the battle of mankind against viruses. The sequencing of this work was supported by China National GeneBank. We appreciate all the authors who have deposited and shared genome data on GISAID, and the genome sequences are acknowledged in Additional file 3 Table S7.

\section{Authors' contributions}

J.L., W.C., and M.X. conceived the project. X.L., J. Z, Y.W., and Y.L. sampled and processed the clinical specimen. M.X., Ji.L., M.L., and J.L. designed the experiments. L.Y. and Y.Z. developed the multiplex PCR amplicon-based sequencing method. M.L., Ji.L., Y. L, P.R. W.S., G.Y., and T.C. performed the multiplex PCR and amplicon sequencing. Ji.L. and P.R. performed the metatranscriptomic library construction and hybrid capture experiments. J.J., M. L, W.S., T.L., H.R., and H.Z. processed the sequencing data and conducted the bioinformatic analyses. J.L., M.X. H.Z., J.J., M.L., and W.S. interpreted the data. M.X., J.J., M.L., and J.L. wrote and polished the manuscript. H.Z., W.S., L.Y., W.C., and Y.Z. contributed substantially to the manuscript revisions. All other authors provided useful suggestions and comments on the project and the manuscript. All authors read and approved the final manuscript.

\section{Funding}

This work is funded by the National Science and Technology Major Project of China (no. 2017ZX10303406); the National Major Project for Control and Prevention of Infectious Disease in China (2018ZX10301101-004); the emergency grants for prevention and control of SARS-CoV-2 of Ministry of Science and Technology (2020YFC0841400) and Guangdong province, China (2020B111107001, 2020B111108001, 2018B020207013); the Guangdong Provincial Key Laboratory of Genome Read and Write (no. 2017B030301011); the Guangdong Provincial Academician Workstation of BGl Synthetic Genomics (no. 2017B090904014); and the Shenzhen Engineering Laboratory for Innovative Molecular Diagnostics (DRC-SZ [2016]884).

\section{Availability of data and materials}

The data that support the findings of this study have been deposited into CNSA (CNGB Nucleotide Sequence Archive, https://db.cngb.org/cnsa/) of CNGBdb with project IDs CNP0000951 [50] and CNP0000955 [51]; into GISAID (https://www.gisaid.org/, registration required) with accession numbers EPI_ISL_414663, EPI_ISL_414686, EPI_ISL_414687, EPI_ISL_414688, EPI_ISL_414689, EPI_ISL_414690, EPI_ISL_414691, and EPI_ISL_414692 [52]; and into NCBI with accession numbers MT568634, MT568635, MT568636, MT568637, MT568638, MT568639, MT568640, MT568641 [53] and PRJNA637515 [54]. The software and parameters used in data analyses during the current study are summarized in Additional file 2 Table S6.

\section{Ethics approval and consent to participate}

Informed consent was obtained from all participants enrolled in studies at the First Affiliated Hospital of Guangzhou Medical University. In addition, the study conformed to the principles of the Declaration of Helsinki. The IRB of BGI-Shenzhen approved the sequencing and downstream analyses of samples collected by the aforementioned institution under ethical clearance no. BGI-IRB 20008

\section{Consent for publication}

Not applicable

\section{Competing interests}

L.Y., Y.Z., F.C., and X.X. have applied for a patent relating to the ampliconbased method, and the details can be found below: PCR primer pair and application thereof

Patent applicant: MGI Tech Co., Ltd Name of inventor(s): Lin Yang, Ya Gao, Guodong Huang, Yicong Wang, Yuqian Wang, Yanyan Zhang, Fang Chen, Na Zhong, Hui Jiang, Xun Xu Application number: PCT/CN2017/089195

The remaining authors declare that they have no competing interests.

\section{Author details}

${ }^{1}$ BGI-Shenzhen, Shenzhen 518083, China. ${ }^{2}$ Shenzhen Key Laboratory of Unknown Pathogen Identification, BGI-Shenzhen, Shenzhen 518083, China. ${ }^{3}$ State Key Laboratory of Respiratory Disease, National Clinical Research Center for Respiratory Disease, Guangzhou Institute of Respiratory Health, the First Affiliated Hospital of Guangzhou Medical University, Guangzhou, China. ${ }^{4}$ School of Future Technology, University of Chinese Academy of Sciences, Beijing 101408, China. ${ }^{5}$ BGl Education Center, University of Chinese Academy of Sciences, Shenzhen 518083, China. ${ }^{6}$ MGl, BGI-Shenzhen, Shenzhen 518083, China. ${ }^{7}$ Institute of Infectious Disease, Guangzhou Eighth People's Hospital of Guangzhou Medical University, Guangzhou, China. ${ }^{8}$ School of Biology and Biological Engineering, South China University of Technology, Guangzhou, China. ${ }^{9}$ BGI PathoGenesis Pharmaceutical Technology, Shenzhen, China. ${ }^{10}$ James D. Watson Institute of Genome Science, Hangzhou 310008, China. ${ }^{11}$ Guangdong Provincial Key Laboratory of Genome Read and Write, BGI-Shenzhen, Shenzhen 518120, China. ${ }^{12}$ Shenzhen Engineering Laboratory for Innovative Molecular Diagnostics, BGI-Shenzhen, Shenzhen 518120, China. 
Received: 27 March 2020 Accepted: 10 June 2020

Published online: 30 June 2020

\section{References}

1. WHO: Coronavirus disease (COVID-2019) situation report - 54. World Health Organization; 2020

2. Dudas G, Carvalho LM, Bedford T, Tatem AJ, Baele G, Faria NR, Park DJ, Ladner JT, Arias A, Asogun D, et al. Virus genomes reveal factors that spread and sustained the Ebola epidemic. Nature. 2017:544:309-15.

3. Dudas G, Carvalho LM, Rambaut A, Bedford T. Correction: MERS-CoV spillover at the camel-human interface. Elife. 2018;7.

4. Gardy JL, Loman NJ. Towards a genomics-informed, real-time, global pathogen surveillance system. Nat Rev Genet. 2018;19:9-20.

5. Gire SK, Goba A, Andersen KG, Sealfon RS, Park DJ, Kanneh L, Jalloh S, Momoh M, Fullah M, Dudas G, et al. Genomic surveillance elucidates Ebola virus origin and transmission during the 2014 outbreak. Science. 2014;345: 1369-72.

6. Grubaugh ND, Ladner JT, Kraemer MUG, Dudas G, Tan AL, Gangavarapu K, Wiley MR, White S, Theze J, Magnani DM, et al. Genomic epidemiology reveals multiple introductions of Zika virus into the United States. Nature. 2017:546:401-5

7. Sabir JS, Lam T, Ahmed MM, Li L, Shen Y, Abo-Aba SE, Qureshi Ml, AbuZeid M, Zhang Y, Khiyami MA, et al. Co-circulation of three camel coronavirus species and recombination of MERS-CoVs in Saudi Arabia. Science. 2016;351:81-4.

8. Lu R, Zhao X, Li J, Niu P, Yang B, Wu H, Wang W, Song H, Huang B, Zhu N, et al. Genomic characterisation and epidemiology of 2019 novel coronavirus: implications for virus origins and receptor binding. Lancet. 2020;395:565-74

9. Zhu N, Zhang D, Wang W, Li X, Yang B, Song J, Zhao X, Huang B, Shi W, Lu $R$, et al. A novel coronavirus from patients with pneumonia in China, 2019. N Engl J Med. 2020;382:727-33.

10. Zhou P, Yang XL, Wang XG, Hu B, Zhang L, Zhang W, Si HR, Zhu Y, Li B, Huang $C L$, et al. A pneumonia outbreak associated with a new coronavirus of probable bat origin. Nature. 2020:579:270-3

11. Wu F, Zhao S, Yu B, Chen YM, Wang W, Song ZG, Hu Y, Tao ZW, Tian JH, Pei $Y Y$, et al. A new coronavirus associated with human respiratory disease in China. Nature. 2020.

12. Drosten C, Chiu LL, Panning M, Leong HN, Preiser W, Tam JS, Gunther $S_{\text {, }}$ Kramme S, Emmerich P, Ng WL, et al. Evaluation of advanced reverse transcription-PCR assays and an alternative PCR target region for detection of severe acute respiratory syndrome-associated coronavirus. J Clin Microbiol. 2004:42:2043-7.

13. Jonsdottir HR, Dijkman R. Coronaviruses and the human airway: a universal system for virus-host interaction studies. Virol J. 2016;13:24.

14. Chan JF, Yuan S, Kok KH, To KK, Chu H, Yang J, Xing F, Liu J, Yip CC, Poon RW, et al. A familial cluster of pneumonia associated with the 2019 novel coronavirus indicating person-to-person transmission: a study of a family cluster. Lancet. 2020;395:514-23.

15. Holshue ML, DeBolt C, Lindquist S, Lofy KH, Wiesman J, Bruce H, Spitters C, Ericson K, Wilkerson S, Tural A, et al. First case of 2019 novel coronavirus in the United States. N Engl J Med. 2020(382):929-36.

16. McCrone JT, Woods RJ, Martin ET, Malosh RE, Monto AS, Lauring AS Stochastic processes constrain the within and between host evolution of influenza virus. Elife. 2018;7:e35962.

17. Ni M, Chen C, Qian J, Xiao HX, Shi WF, Luo Y, Wang HY, Li Z, Wu J, Xu PS, et al. Intra-host dynamics of Ebola virus during 2014. Nat Microbiol. 2016;1:16151.

18. Park DJ, Dudas G, Wohl S, Goba A, Whitmer SL, Andersen KG, Sealfon RS, Ladner JT, Kugelman JR, Matranga CB, et al. Ebola virus epidemiology, transmission, and evolution during seven months in Sierra Leone. Cell. 2015;161:1516-26.

19. Domingo E, Sheldon J, Perales C. Viral quasispecies evolution. Microbiol Mol Biol Rev. 2012;76:159-216.

20. Wood DE, Salzberg SL. Kraken: ultrafast metagenomic sequence classification using exact alignments. Genome Biol. 2014;15:R46.

21. Chen S, Zhou Y, Chen Y, Gu J. fastp: an ultra-fast all-in-one FASTQ preprocessor. Bioinformatics. 2018;34:1884-90.

22. Chen Y, Chen Y, Shi C, Huang Z, Zhang Y, Li S, Li Y, Ye J, Yu C, Li Z, et al. SOAPnuke: a MapReduce acceleration-supported software for integrated quality control and preprocessing of high-throughput sequencing data. Gigascience. 2018;7:1-6.
23. Schmieder R, Edwards R. Quality control and preprocessing of metagenomic datasets. Bioinformatics. 2011;27:863-4.

24. Au CH, Ho DN, Kwong A, Chan TL, Ma ESK. BAMClipper: removing primers from alignments to minimize false-negative mutations in amplicon nextgeneration sequencing. Sci Rep. 2017;7:1567.

25. Bankevich A, Nurk S, Antipov D, Gurevich AA, Dvorkin M, Kulikov AS, Lesin VM, Nikolenko SI, Pham S, Prjibelski AD, et al. SPAdes: a new genome assembly algorithm and its applications to single-cell sequencing. J Comput Biol. 2012;19:455-77.

26. Walker BJ, Abeel T, Shea T, Priest M, Abouelliel A, Sakthikumar S, Cuomo CA, Zeng Q, Wortman J, Young SK, Earl AM. Pilon: an integrated tool for comprehensive microbial variant detection and genome assembly improvement. PLoS One. 2014:9:e112963.

27. Tan W, Zhao X, Wang W, Ma X, Jiang Y, Lu R, Wang J, Zhou W, Niu P, Liu P, et al. hCoV-19/Wuhan/IVDC-HB-01/2019. GISAID Accession: EPI ISL 402119. GISAID EpiCOV. https://www.epicov.org/epi3/. Accessed 10 Jan 2020.

28. Li H, Durbin R. Fast and accurate short read alignment with BurrowsWheeler transform. Bioinformatics. 2009;25:1754-60.

29. Li H, Handsaker B, Wysoker A, Fennell T, Ruan J, Homer N, Marth G, Abecasis G, Durbin R. 1000 Genome Project Data Processing Subgroup. The sequence alignment/map format and SAMtools. Bioinformatics. 2009:25:2078-9.

30. Wickham H: ggplot2. Wiley Interdisciplinary Reviews: Computational Statistics 2011, 3:180-185.

31. R Core Team. R: A language and environment for statistical computing. $R$ Foundation for Statistical Computing, Vienna, Austria. https://www.R-project. org/. Accessed 5 July 2019

32. Kolde R. pheatmap: Pretty Heatmaps. https://www.rdocumentation.org/ packages/pheatmap. Accessed 4 Jan 2019.

33. Kassambara A. ggpubr:"ggplot2" based publication ready plots. https:// rpkgs.datanovia.com/ggpubr. Accessed 13 Feb 2020

34. Garrison E, Marth G. Haplotype-based variant detection from short-read sequencing. arXiv:1207.3907v2 [q-bio.GN]. Accessed 20 July 2012.

35. Seemann T. Snippy: fast bacterial variant calling from NGS reads. https:// github.com/tseemann/snippy. Accessed 28 Nov 2017.

36. Cingolani P, Platts A, Wang le L, Coon M, Nguyen T, Wang L, Land SJ, Lu X, Ruden DM. A program for annotating and predicting the effects of single nucleotide polymorphisms, SnpEff: SNPs in the genome of Drosophila melanogaster strain w1118; iso-2; iso-3. Fly (Austin). 2012;6(2):80-92.

37. Li R, Yu C, Li Y, Lam TW, Yiu SM, Kristiansen K, Wang J. SOAP2: an improved ultrafast tool for short read alignment. Bioinformatics. 2009:25:1966-7.

38. Kim D, Paggi JM, Park C, Bennett C, Salzberg SL. Graph-based genome alignment and genotyping with HISAT2 and HISAT-genotype. Nat Biotechnol. 2019;37:907-15.

39. Wood DE, Lu J, Langmead B. Improved metagenomic analysis with Kraken 2. Genome Biol. 2019:20:257.

40. Lu J, Breitwieser FP, Thielen P, Salzberg SL. Bracken: estimating species abundance in metagenomics data. PeerJ Computer Science. 2017:3:e104.

41. Li Q, Zhao X, Zhang W, Wang L, Wang J, Xu D, Mei Z, Liu Q, Du S, Li Z, et al. Reliable multiplex sequencing with rare index mis-assignment on DNBbased NGS platform. BMC Genomics. 2019;20:215.

42. Xia Z, Jiang Y, Drmanac R, Shen H, Liu P, Li Z, Chen F, Jiang H, Shi S, Xi Y. Advanced whole genome sequencing using a complete PCR-free massively parallel sequencing (MPS) workflow. bioRxiv. 2019.12.20.885517. https://doi. org/10.1101/2019.12.20.885517.

43. Kircher M, Sawyer S, Meyer M. Double indexing overcomes inaccuracies in multiplex sequencing on the Illumina platform. Nucleic Acids Res. 2012;40: e3.

44. Metsky HC, Matranga CB, Wohl S, Schaffner SF, Freije CA, Winnicki SM, West K, Qu J, Baniecki ML, Gladden-Young A, et al. Zika virus evolution and spread in the Americas. Nature. 2017;546:411-5.

45. Zou L, Ruan F, Huang M, Liang L, Huang H, Hong Z, Yu J, Kang M, Song Y, Xia J, et al. SARS-CoV-2 viral load in upper respiratory specimens of infected patients. N Engl J Med. 2020;382:1177-9.

46. Chan KH, Poon LL, Cheng VC, Guan Y, Hung IF, Kong J, Yam LY, Seto WH, Yuen KY, Peiris JS. Detection of SARS coronavirus in patients with suspected SARS. Emerg Infect Dis. 2004:10:294-9.

47. Mohran KA, Farag EA, Reusken CB, Raj VS, Lamers MM, Pas SD, Voermans J, Smits SL, Alhajri MM, Alhaji F, et al. The sample of choice for detecting Middle East respiratory syndrome coronavirus in asymptomatic dromedary camels using real-time reversetranscription polymerase chain reaction. Rev Sci Tech. 2016;35:905-11. 
48. Quick J, Grubaugh ND, Pullan ST, Claro IM, Smith AD, Gangavarapu K, Oliveira G, Robles-Sikisaka R, Rogers TF, Beutler NA, et al. Multiplex PCR method for MinION and Illumina sequencing of Zika and other virus genomes directly from clinical samples. Nat Protoc. 2017:12:1261-76.

49. Quick J. nCoV-2019 sequencing protocol v2. https://doi.org/10.17504/ protocols.io.bdp7i5rn. Accessed 9 Apr 2020.

50. Zhao J, Wang Y. COVID-19-Project-1-the First Affiliated Hospital of Guangzhou Medical University. CNGB Project ID: CNP0000951. https://db. cngb.org/search/project/CNP0000951/. CNGB Sequence Archive. Accessed 16 Mar 2020.

51. Ji J. Multiple approaches for massively parallel sequencing of HCoV-19 samples. CNGB Project ID: CNP0000955. CNGB Sequence Archive. https://db. cngb.org/search/project/CNP0000955/. Accessed 3 Apr 2020.

52. Zhao J, Wang Y. hCoV-19/Guangzhou. GISAID Accession: EPI_ISL_414663, EPI_ISL_414686-EPI_ISL_414692. GISAID EpiCOV. https://www.epicov.org/ epi3/. Accessed 16 Mar 2020.

53. Zhao J, Wang Y, Li M. Multiple approaches for massively parallel sequencing of HCoV-19 (SARS-CoV-2) genomes directly from clinical samples. GenBank: MT568634-MT568641. GenBank. https://www.ncbi.nlm.nih.gov/nuccore/. Accessed 5 June 2020

54. Ji J. Multiple approaches for massively parallel sequencing of SARS-CoV-2 samples. BioProject Accession: PRJNA637515. NCBI Sequence Read Archive. https://www.ncbi.nlm.nih.gov/bioproject/PRJNA637515. Accessed 5 June 2020

\section{Publisher's Note}

Springer Nature remains neutral with regard to jurisdictional claims in published maps and institutional affiliations.

Ready to submit your research? Choose BMC and benefit from:

- fast, convenient online submission

- thorough peer review by experienced researchers in your field

- rapid publication on acceptance

- support for research data, including large and complex data types

- gold Open Access which fosters wider collaboration and increased citations

- maximum visibility for your research: over $100 \mathrm{M}$ website views per year

At BMC, research is always in progress.

Learn more biomedcentral.com/submissions 